\title{
Inter-Layer FEC Aided Unequal Error Protection for Multi-Layer Video Transmission in Mobile TV
}

\author{
Yongkai Huo, Mohammed El-Hajjar and Lajos Hanzo, Fellow, IEEE \\ School of ECS, University of Southampton, UK. Fax: +44-23-8059 4508 \\ Email: $\{y h 3 g 09$, meh, lh $\} @$ ecs.soton.ac.uk, http://www-mobile.ecs.soton.ac.uk
}

\begin{abstract}
Layered video coding creates multiple layers of unequal importance, which enables us to progressively refine the reconstructed video quality. When the base layer $(\mathrm{BL})$ is corrupted or lost during transmission, the enhancement layers (ELs) must be dropped, regardless whether they are perfectly decoded or not, which implies that the transmission power assigned to the ELs is wasted. In this treatise, we propose an interlayer forward error correction (FEC) coded video transmission scheme for mobile TV. At the transmitter, the proposed interlayer (IL) coding technique implants the systematic information of the BL into the ELs by using exclusive-OR operations. At the receiver, the implanted bits of the ELs may be utilized for assisting in decoding the BL. Furthermore, the data partition mode of $\mathrm{H} .264$ video coding is utilized as the source encoder, where the type $B$ and type $C$ partitions will assist in protecting the type A partition. The IL coded bitstream will then be modulated and transmitted over a multi-functional multiple-input multiple output (MF-MIMO) scheme for the sake of improving the system's performance in mobile environments. The proposed system may be readily combined with the traditional unequal error protection (UEP) technique, where extrinsic mutual information (MI) measurements are used for characterizing the performance of our proposed technique. Finally, our simulation results show that the proposed system model outperforms the traditional UEP aided system by about $2.5 \mathrm{~dB}$ of $E_{b} / N_{0}$ or $3.4 \mathrm{~dB}$ of peak signal-to-noise ratio (PSNR) at the cost of $21 \%$ complexity increase, when employing a recursive systematic convolutional code. Furthermore, unlike the traditional UEP strategies, where typically stronger FEC-protection is assigned to the more important layer, employing our proposed IL coding technique requires weaker FEC to the more important layer. For example, the system relying on channel coding rates of 0.85 , 0.44 and 0.44 for the type A, type $B$ and type C $\mathbf{H} .264$ video partitions, respectively, achieves the best system performance when employing a recursive systematic convolutional (RSC) code.
\end{abstract}

\section{INTRODUCTION}

Layered video coding [1] was proposed and has been adopted by a number of existing video coding standards [2]-[5], which is capable of generating multiple layers of unequal importance. Generally the most important layer and the less important layers are referred to as the base layer (BL) and enhancement layers (ELs), respectively. A multiview profile (MVP) [2] was developed by the moving picture expert group (MPEG)'s [6] video coding standard, where the left view and right view were encoded into a BL and an EL, respectively. Another layered video coding standard referred to as scalable video coding (SVC) [3], [4] was recently developed

Copyright (c) 2013 IEEE. Personal use of this material is permitted. However, permission to use this material for any other purposes must be obtained from the IEEE by sending an email to pubs-permissions@iee.org.

The financial support of the EU's Concerto project, of the European Research Council's Senior Fellow Grant, of the EPSRC under the auspices of the China-UK Science Bridge and that of the RC-UK under the India-UK Advanced Technology Centre (IU-ATC) is gratefully acknowledged. as an extension of H.264/AVC [4], which encodes a video sequence into multiple layers, where a reduced-size subset of the bitstream may be extracted to meet the users' specific preferences. Moreover, the less important layers have lower priority and hence may be dropped in the transmission scenario of network congestion or buffer overflow [7]. In layered video transmission relying on SVC [3] streaming for example, when the $\mathrm{BL}$ is corrupted or lost due to channel impairments, the ELs must also be dropped by the video decoder even if they are perfectly received.

Unequal error protection (UEP) was firstly proposed by Masnick and Wolf in [8], which allocates stronger forward error correction (FEC) to the more important data, while dedicating weaker FEC to the less important video parameters. Since then numerous UEP techniques have proposed. A novel UEP modulation concept was investigated in [9] for the specific scenarios, where channel coding cannot be employed. Hence UEP was achieved by allocating different transmission power to individual bits according to their bit error sensitivity albeit in practice this remains a challenge. Additionally, the UEP capabilities of convolutional codes (CC) were studied in [10], while rate-compatible convolutional codes (RCPC) were proposed by Hagenauer [11]. Furthermore, as a benefit of the outstanding performance of low-density parity-check (LDPC) codes, a number of UEP design methodologies [12][15] have been investigated using LDPC codes. The socalled UEP density evolution (UDE) technique of [12], [15] was proposed for transmission of video streams over binary erasure channels (BEC). The authors of [13] proposed a new family of UEP codes, based on LDPC component codes, where the component codes are decoded iteratively in multiple stages, while the order of decoding and the choice of the LDPC component codes jointly determine the level of error protection. A practical UEP scheme using LDPC codes was proposed in [14], where the high-significance bits were more strongly protected than low-significance bits.

However, most of the above UEP studies considered artificially generated signals of unequal significance, rather than realistic video signals. Naturally, the significance differentiation of practical video signals is more challenging. In compressed video streams, as in layered video coding, different bits may have different significance. Therefore, again it is intuitive to employ UEP for protecting the more important bits by stronger FEC codecs than the less important bits, in order to achieve an improved reconstructed video quality. Nonetheless, a number of contributions have been made also in the field of UEP video communications relying on realistic video signals. For example, an UEP scheme was conceived in [16] for objectbased video communications for achieving the best attainable video quality under specific bitrate and delay constraints in an error-prone network environment. A jointly optimized 
turbo transceiver capable of providing UEP for wireless video telephony was proposed in [17]. The performance of datapartitioning [4] H.264/AVC video streaming using recursive systematic convolutional (RSC) codes aided UEP was evaluated in [18]. In [19], UEP based turbo coded modulation was investigated, where both the channel capacity and the cutoff rates of UEP levels were determined. A novel UEP method was proposed in [20] for SVC video transmission over networks subject to packet-loss events. Firstly, the authors presented an efficient performance metric, termed as the layerweighted expected zone of error propagation (LW-EZEP), for quantifying the error propagation effects imposed by packet loss events. A novel UEP scheme was proposed in [21], which considered the unequal importance of the frames in a GOP, as well as that of the macroblocks in a video frame. An efficient FEC-coded scheme was also proposed by Chang et al. in [21]. They also considered the different importance of the intra-coded (I) frame and of the predicted (P) frames within a group of pictures (GOP) [22]. The video bits of different importance were mapped to the different-protection bits of the modulation constellation points with the assistance of hierarchical quadrature amplitude modulation (QAM). The authors of [23] proposed cross-layer operation aided scalable video streaming, which aimed for the robust delivery of the SVC-coded video stream over error-prone channels. The video distortion endured was first estimated based on both the available bandwidth and the packet loss ratio (PLR) experienced at the transmitter. The achievable video quality was then further improved with the aid of content-aware bitrate allocation and a sophisticated bit detection technique was conceived, which took into account the estimated video distortion. Finally, a powerful error concealment method was invoked at the receiver. An UEP scheme using Luby Transform (LT) codes was developed in [24] for the sake of recovering the video packets dropped at the routers, owing to tele-traffic congestions, noting that the high delay of LT codecs is only applicable to delay-tolerant broadcast-style video streaming services.

In the traditional UEP schemes conceived for layered video communication, variable-rate FEC was invoked for the different layers. When the BL is corrupted or lost, the ELs also have to be dropped, regardless whether they are perfectly received or not, which implies that the transmission power assigned to the ELs was wasted. The so-called layer-aware FEC (LA-FEC) philosophy [25], [26] using a Raptor codec was invoked for video transmission over the BEC. At the transmitter, the channel encoding was performed right across the BL and the ELs. As a benefit, at the receiver, the parity bits of the ELs may be additionally invoked for assisting in correcting the errors within the BL. Motivated by these advances, we developed an inter-layer operation aided FEC (IL-FEC) scheme relying on a systematic FEC code in [27], where the systematic bits of the BL were implanted into the ELs. At the receiver, the above-mentioned implanted bit of the ELs may be utilized for assisting in decoding the BL. The IL-FEC technique of [27] was also combined with the UEP philosophy for the sake of further improving the attainable system performance. Our proposed technique is significantly different with the LA-FEC philosophy proposed in [25], [26], as detailed below conceiving the following aspects. Firstly, our technique is proposed for layered video communication over wireless channels, while the LA-FEC of [25], [26] is proposed for the BEC. Secondly, IL-FEC invokes the soft decoding aided channel codecs, such as an RSC code, while the LA-FEC of [25], [26] considered a hard-decoding based Raptor codec. In this context, we note that Raptor codes are less suitable for low-delay lip-synchronized interactive multimedia communications, whilst our scheme is readily applicable. Furthermore, it is important to note that the LAFEC cannot be readily applied in soft decoding aided channel codecs. Finally, IL-FEC implants the systematic bits of the BL into the ELs, while the LA-FEC [25], [26] generates the parity bits across the BL and ELs.

At the time of writing, multimedia content is evolving from traditional content to a range of rich, heterogeneous media content, such as traditional TV, streaming audio and video as well as image and text messaging. Furthermore, in the current era of smart phones, mobile TV has become an appealing extension of terrestrial TV. Additionally, in order to meet the challenging performance requirements in bandwidth-constrained environments, multiple input multiple output (MIMO) systems constitute a promising transmission solution. Layered steered space-time codes (LSSTC) [28], [29] combine the benefits of the vertical Bell Labs space-time (VBLAST) scheme [30], of space-time block codes [31] and of beamforming [32]. Hence LSSTCs are invoked for providing both a diversity gain to achieve a high BER performance in mobile environments as well as for attaining a multiplexing gain in order to maintain a high data rate. In this treatise, we propose a system for transmitting an IL-FEC encoded compressed video bitstream with the aid of a LSSTC transceiver structure (IL-FEC-LSSTC) for mobile TV broadcasting. This scheme may be considered as an evolution of the traditional UEP schemes exemplified by [20], [23]. The data partitioning mode (PM) of the H.264 video codec is employed, where the type $\mathrm{B}$ and type $\mathrm{C}$ partitions will be utilized for protecting the type A partition ${ }^{1}$. The mutual information (MI) at the output of the FEC decoder is measured [33] for the sake of analyzing the performance of our proposed system. Finally, differentrate, different-protection channel codecs will be employed as FEC codes for improving the attainable system performance.

Against this background, the main rationale and novelty of this paper can be summarized as follows. We conceive an inter-layer FEC codec for layered video streaming, which is combined with cutting-edge UEP and LSSTC schemes for the sake of improving the attainable mobile TV performance with the aid of mutual information analysis. Additionally, the following conclusions transpire from our investigations:

1) Only a modest complexity increase is imposed by our inter-layer protection technique, which guarantees the practical feasibility of our proposed technique. Specifically, 21\% complexity increase is imposed by our interlayer decoding technique, when employing a RSC codec.

2) Intriguingly, we found that in the context of employing our proposed technique, the more important layer should be protected by less FEC-redundancy to achieve the best overall system performance for H.264/AVC partitioning mode aided compressed video streaming, which is un-

${ }^{1}$ For brevity, we will often simply refer to them as A, B and C 
expected in the light of the traditional unequal error protection strategy. For example, the system relying on the channel coding rates of $0.85,0.44$ and 0.44 for the $A$, $B$ and C H.264/AVC partitions, respectively, achieves the best system performance when employing a RSC code for the transmission of the Football sequence.

Again, we use the H.264/AVC data partitioning mode in our simulations, but our proposed scheme is not limited to partitioning based video, it may be readily applied in any arbitrary system relying on layered video coding, such as scalable video coding [34]. The rest of this paper is organized as follows. In Section II, we briefly review the state-of-the-art layered video techniques. Section III details our proposed ILFEC-LSSTC system model and the related video transmission techniques. Then the performance of our proposed system is analyzed using mutual information in Section IV. The performance of our IL-FEC-LSSTC scheme using a RSC codec is benchmarked in Section V using two video sequences having different motion characteristics. Finally, we offer our conclusions in Section VI.

\section{LAYERED Video STREAMING}

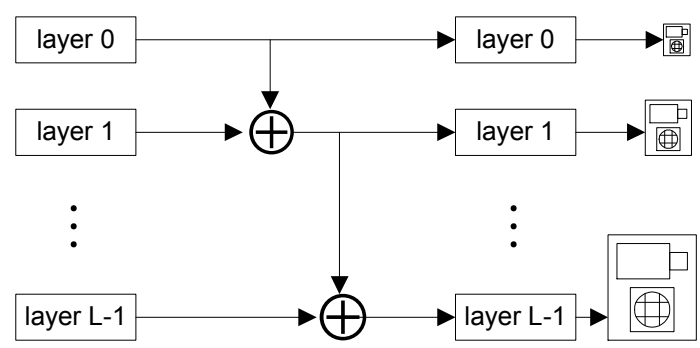

Fig. 1. Architecture of a layered video scheme [1], where the video quality is refined progressively.

Layered video compression [1], [3], [26] encodes a video sequence into multiple layers, which enable us to progressively refine the reconstructed video quality at the receiver. Generally, the most important layer is referred to as the BL, which may be relied upon by multiple ELs. Furthermore, an EL may be further relied upon by other ELs. Again, when the BL or an EL is lost or corrupted during its transmission, the dependent layers cannot be utilized by the decoder and must be dropped. A layered video scheme is displayed in Fig. 1, where layer $i$ $(0<i \leq L-1)$ depends on layer $(i-1)$ for decoding, while layer $i$ improves the video quality of layer $(i-1)$.

The subject of SVC [3] has been an active research field for over two decades. This terminology is also used in the Annex $\mathrm{G}$ extension of the H.264/AVC video compression standard [4]. Indeed, SVC is capable of generating several bitstreams that may be decoded at a similar quality and compression ratio to that of the existing H.264/AVC codec. When for example low-cost, low-quality streaming is required by the users, some of the ELs may be removed from the compressed video stream, which facilitates flexible bitrate-control based on the specific preferences of the users.

Recently, the Joint Video Team (JVT) proposed multiview video coding (MVC) as an amendment to the H.264/AVC standard [4]. Apart from the classic techniques employed in single-view coding, multiview video coding invokes the socalled inter-view correction technique by jointly processing the different views for the sake of reducing the bitrate. Hence, the first encoded view may be termed as the BL, while the remaining views may be treated as the ELs.

A number of layered video coding schemes have been developed and some of them are adopted by recent video coding standards, for example the scalable video coding [3] and data partitioning (DP) [4], [35], [36]. In this treatise, we use data partitioning based layered video coding in our simulations, which is a beneficial feature of the H.264/AVC codec [4]. In the data partitioning mode, the data streams representing different semantic importance are categorized into a maximum of three bitstreams/partitions [37] per video slice, namely type $\mathrm{A}$, type $\mathrm{B}$ and type $\mathrm{C}$ partitions. The header information, such as macroblock (MB) types, quantization parameters and motion vectors are carried by the A partition. The B partition is also referred to as the intra-frame-coded partition, which contains intra-frame-coded information, including the coded block patterns (CPBs) and intra-frame coded coefficients. The $\mathrm{B}$ partition is capable of prohibiting error propagation in the scenario, when the reference frame of the current motioncompensated frame is corrupted. In contrast to the B partition, the $\mathrm{C}$ partition is the inter-frame-coded partition, which carries the inter-CBPs and the inter-frame coded coefficients. The $\mathrm{C}$ partition has to rely on the reference frame for reconstructing the current picture. Hence, if the reference picture is corrupted, errors may be propagated to the current frame. Amongst these three partitions, the type A partition may be deemed to be the most important one, which may be treated as the BL. Correspondingly, the $\mathrm{B}$ and $\mathrm{C}$ partitions may be interpreted as a pair of ELs, since they are dependent on the A partition for decoding. Albeit the information in partitions $\mathrm{B}$ and $\mathrm{C}$ cannot be used in the absence of A, partition B and $\mathrm{C}$ can be used independently of each other, again, given the availability of A. In this treatise, we will employ the partitioning mode of H.264/AVC for benchmarking our system.

\section{SySTEM OVERVIEW}

In this section, we will briefly introduce the architecture of the inter-layer FEC scheme [27] conceived for layered video transmission over our LSSTC scheme for mobile TV transmission. The system's structure is displayed in Fig. 2, where data-partitioning aided H.264 [4] encoding and LSSTC transmission are employed, while the structures of the variable node decoder (VND) and check node decoder (CND) [38] are further detailed in Fig. 3. Both the VND and CND blocks may accept a maximum of three soft information inputs and generate a maximum of three soft information outputs with the goal of iteratively exploiting all IL dependencies amongst the FEC coded layers A, B and C. Specifically, assuming that $u_{1}$, $u_{2}$ and $u_{3}=u_{1} \oplus u_{2}$ are random binary variables, the action of the VND of Fig. 3 sums two LLR inputs for generating a more reliable LLR output, which may be formulated as $L_{o_{3}}\left(u_{1}\right)=L_{i_{1}}\left(u_{1}\right)+L_{i_{2}}\left(u_{1}\right)$. The boxplus operation of $L\left(u_{3}=u_{1} \oplus u_{2}\right)=L\left(u_{1}\right) \boxplus L\left(u_{2}\right)$ [39] may be utilized for deriving the confidence of the bit $u_{3}$, given that the confidence of the bits $u_{1}$ and $u_{2}$ is known. Specifically, the boxplus 


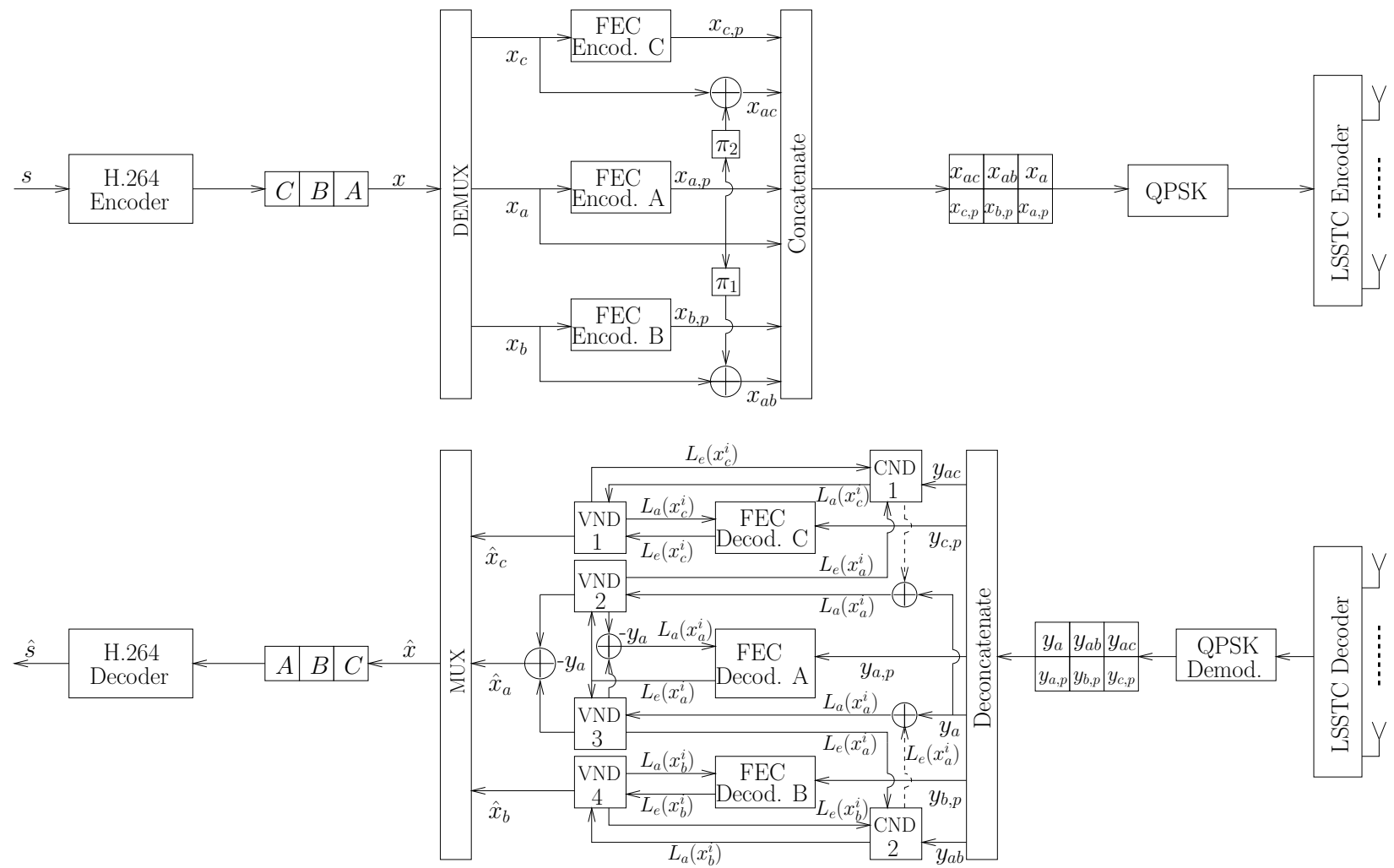

Fig. 2. IL-FEC encoding architecture of H.264 data partitioning mode coded video.

operation $\boxplus$ is defined as follows [40]

$$
\begin{aligned}
& L\left(u_{1}\right) \boxplus L\left(u_{2}\right)=\log \frac{1+e^{L\left(u_{1}\right)} e^{L\left(u_{2}\right)}}{e^{L\left(u_{1}\right)}+e^{L\left(u_{2}\right)}} \\
& =\operatorname{sign}\left[L\left(u_{1}\right)\right] \cdot \operatorname{sign}\left[L\left(u_{2}\right)\right] \cdot \min \left[\left|L\left(u_{1}\right)\right|,\left|L\left(u_{2}\right)\right|\right] \\
& +\log \left[1+e^{-\left|L\left(u_{1}\right)+L\left(u_{2}\right)\right|}\right]-\log \left[1+e^{-\left|L\left(u_{1}\right)-L\left(u_{2}\right)\right|}\right] .
\end{aligned}
$$

In contrast to the above-mentioned VND function, the CND operation of Fig. 3 may be formulated as $L_{o}\left(u_{3}\right)=L_{i}\left(u_{1}\right) \boxplus$ $L_{i}\left(u_{2}\right)$ for extracting the confidence of the bit $u_{3}$, given the LLR input of the bits $u_{1}$ and $u_{2}$.

In Section III-A, we first detail the techniques employed at the transmitter. Then, our inter-layer H.264 decoding techniques and the LSSTC receiver will be illustrated in Section III-B, with special emphasis on how the VND and the CND exchange their inter-layer redundancy for improving the overall performance of the system. We assume that $A$ is the BL and $B, C$ are the corresponding dependent layers, but both partition $B$ and $C$ can be utilized for protecting the partition $A$. In Section III-A and III-B, we assume that all the layers $A, B$ and $C$ contain $n$ bits for the sake of convenient explanation, while in Section III-C we extend our algorithm to the more general scenario, where the layers have unequal length. Finally, Section III-D discusses the overheads imposed by our proposed IL technique, including its delay, complexity and its FEC-redundancy.

\section{A. Transmitter Model}

At the transmitter, the video source signal $s$ is compressed using the data partitioning mode of the H.264 encoder, generating partitions $A, B$ and $C$. Then the output bitstream is de-multiplexed into three bitstreams by the DEMUX block of

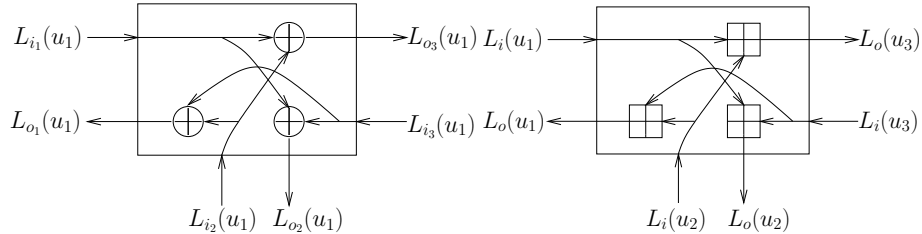

Fig. 3. The structure of VND (left) and CND (right), where $\oplus$ and $\boxplus$ indicate the addition and boxplus operation, respectively. $L_{i}(\cdot)$ and $L_{o}(\cdot)$ indicate the input and output LLR, respectively.

Fig. 2, namely into streams $A, B$ and $C$, carrying the $A, B$ and $C$ partitions of all slices. The resultant binary sequences are $x_{a}, x_{b}$ and $x_{c}$, representing three different layers, as shown in Fig. 2. Then the resultant three layers are encoded as follows:

1) The BL bit sequence $x_{a}$ representing $\mathrm{A}$ will be encoded by the FEC encoder A of Fig. 2, which results in the encoded bits containing the systematic bits $x_{a}$ and parity bits $x_{a, p}$.

2) The bit sequence of the EL $x_{b}$ representing B will firstly be encoded into the systematic bits $x_{b}$ and the parity bits $x_{b, p}$ by the FEC encoder B. Then the XOR operation will be utilized for implanting the systematic information of $x_{a}$ into the systematic information of $x_{b}$ without changing the parity bits of the $\mathrm{B}$ partition $x_{b, p}$. Specifically, the implantation process results in the check bits $x_{a b}^{i}=x_{a}^{i} \oplus x_{b}^{i}$. After this procedure, both the check bits $x_{a b}^{i}$ and the parity bits $x_{b, p}$ are output.

3) Similar to the encoding process of the B partition, the bit sequence of the EL $x_{c}$ representing the $\mathrm{C}$ partition will be encoded into the check bits $x_{a c}^{i}=x_{a}^{i} \oplus x_{c}^{i}$ and the parity bits $x_{c, p}$. 


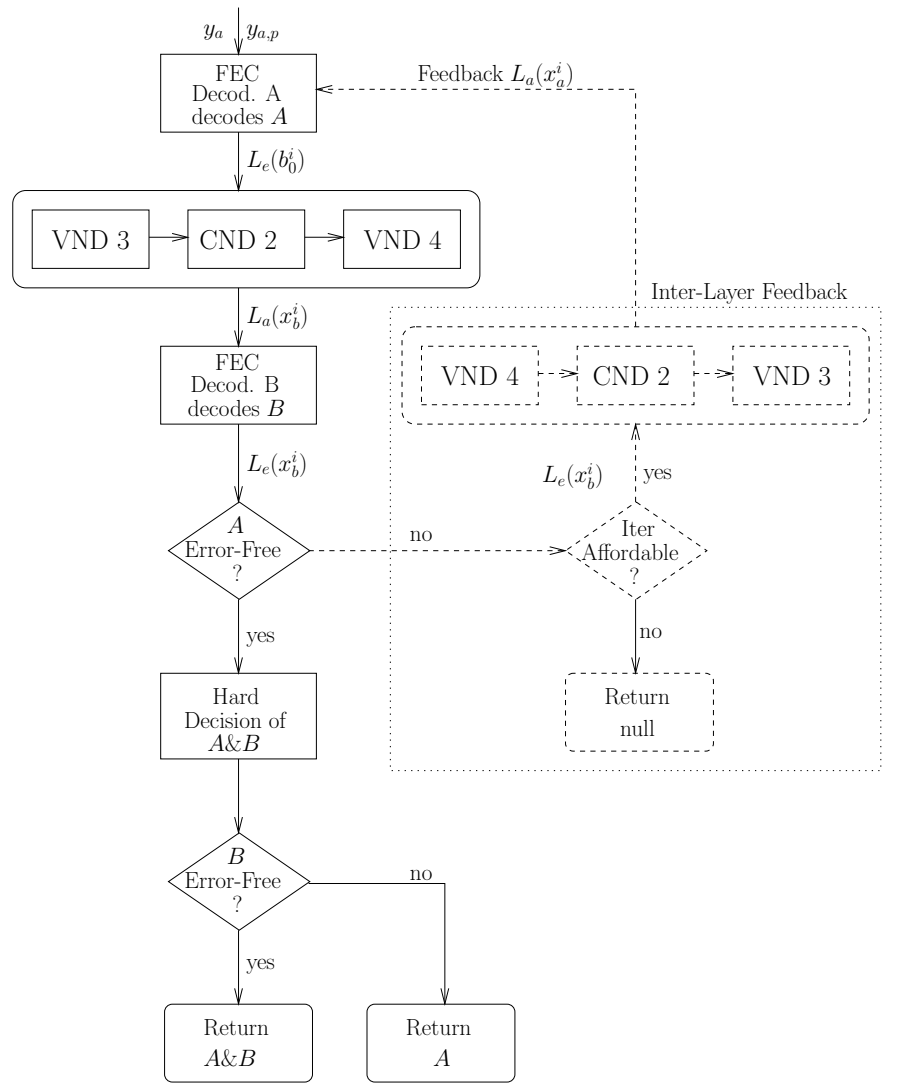

Fig. 4. The flow chart for inter-layer aided FEC decoding of BL $A$ and EL $B$.

Finally, the bit sequences $x_{a}, x_{a, p}, x_{a b}, x_{a, p}, x_{a c}$ and $x_{c, p}$ are concatenated into a joint bitstream for transmission. Note however that the layers $x_{a}$ and $x_{b}, x_{c}$ may contain a different number of bits. Again, the algorithm designed for this scenario will be detailed in Section III-C. Additionally, the interleavers $\pi_{1}$ and $\pi_{2}$ are employed for interleaving the BL $x_{a}$, before its XOR-based implantation into the ELs $x_{b}$ and $x_{c}$.

Following the IL-FEC encoding procedure, the resultant bits are modulated by the quadrature phase-shift keying (QPSK) modulator of Fig. 2 and then transmitted over the LSSTC based MIMO transmitter architecture. Specifically, the transmission structure shown in Fig. 2 has $N_{t}=4$ transmit antennas, which are spaced sufficiently for apart in order to encounter independent fading. The receiver is also equipped with $N_{r}=4$ receive antennas, where the LSSTC system used is characterized by a diversity order of 2 and multiplexing order of 2. Hence the LSSTC used is capable of providing twice the data rate of a single antenna system, while achieving a diversity order of two.

\section{B. Receiver Model}

In this section, we exemplify the IL decoding process using BL $A$ and EL $B$, while the IL decoding process of BL $A$ and EL $C$ is similar. At the receiver ${ }^{2}$, the LSSTC decoding is performed [29]. Then the resultant soft signal will be demodulated by the QPSK demodulator, which generates the log-likelihood ratios (LLR). The LLR information contains the

${ }^{2}$ The deinterleavers $\pi^{-1}$ and $\pi^{-2}$ are ignored at the receiver for the sake of simplifying the system architecture. systematic information $y_{a}, y_{a b}, y_{a c}$ and the parity information $y_{a, p}, y_{b, p}$ and $y_{c, p}$, for the $A, B$ and $C$ partitions, respectively. Following the demodulator, the IL-FEC decoder of Fig. 2 is invoked for exchanging extrinsic information across the three layers. The IL aided FEC decoding process is illustrated by the flow-chart of Fig. 4. Firstly, the FEC decoder A will decode the received information $y_{a}$ and $y_{a, p}$ for estimating the LLRs of the bits $x_{a}$ of the BL $A$. Then, the resultant extrinsic LLR information of $\mathrm{BL} A$ will be input to the "VND3-CND2VND4" block of Fig. 4 for extracting the a-priori LLRs $L_{a}\left(x_{b}^{i}\right)^{3}$ of EL $B$, which is carried out by following the processing of the LLRs in the VND 3, CND 2 and VND 4 components of Fig. 3. Specifically, the "VND3-CND2-VND4" block of Fig. 4 performs the following operations step-by-step:

1) VND 3 generates the information of BL $A$ for CND 2. The inputs to VND 3 block are constituted of the soft information $L_{e}\left(x_{a}^{i}\right)$ generated by the FEC decoder A and the soft information $L_{a}\left(x_{a}^{i}\right)$ generated by summing the channel information $y_{a}$ and $L_{e}\left(x_{a}^{i}\right)$, where $L_{e}\left(x_{a}^{i}\right)$ is generated by CND 2. The output of the VND 3 block is the soft information of $A$. The output can be readily derived as detailed in Fig. 3. The extrinsic LLR $L_{e}\left(x_{a}^{i}\right)$ generated by the FEC decoder $\mathrm{A}$ is input to the VND 3 block of Fig. 2, which extracts the extrinsic LLR information $L_{e}\left(x_{a}^{i}\right)$ and forwards it to the CND 2 block of Fig. 2. Since VND $3^{4}$ has two input branches, it simply duplicates the soft information $L_{e}\left(x_{a}^{i}\right)$.

2) CND 2 generates the information of layer $B$ for VND 4. The inputs of the CND 2 block are the soft check information $y_{a b}$ received from the channel, the soft information $L_{e}\left(x_{a}^{i}\right)$ of $\mathrm{BL} A$ generated by VND 3 and the soft information $L_{e}\left(x_{b}^{i}\right)$ of EL $B$ generated by FEC decoder $B$ of Fig. 2. The output of CND 2 is the soft information of EL $B L_{a}\left(x_{b}^{i}\right)$. The outputs can be readily derived as detailed in Fig. 3. The LLR information $L_{e}\left(x_{a}^{i}\right)$ and the received check information $y_{a b}$ is input to the CND 2 block of Fig. 2 for extracting the LLR information of the systematic bit $x_{b}^{i}$, namely the soft input $L_{a}\left(x_{b}^{i}\right)$ of VND 4 .

3) VND 4 generates the information of EL $B$ for FEC decoder B. The inputs to the VND 4 block are the soft information $L_{a}\left(x_{b}^{i}\right)$ gleaned from CND 2 and the soft information $L_{e}\left(x_{b}^{i}\right)$ generated by FEC decoder B. The output of VND 4 is the soft information of layer $B$. The LLR information $L_{a}\left(x_{b}^{i}\right)$ extracted by the CND 2 is input to the VND 4 block of Fig. 2, which extracts the LLR information $L_{a}\left(x_{b}^{i}\right)$ input to the FEC decoder B of Fig. 2.

Then, the FEC decoder B of Fig. 4 will decode the EL $B$ with the aid of the resultant a-priori LLR $L_{a}\left(x_{b}^{i}\right)$ and of the soft parity information received from the channel, namely $y_{b, p}$ of Fig. 2. Afterwards, the classic cyclic redundancy check (CRC) is invoked for detecting, whether the recovered $\mathrm{BL} A$ is errorfree or not, as shown in Fig. 4. This check results in two

\footnotetext{
${ }^{3}$ As usual, the subscripts "a" and "e" in $L_{a}$ and $L_{e}$ stand for the apriori information and extrinsic information [41], respectively.

${ }^{4}$ All the VNDs of Fig. 2 have two input branches and three output branches, resulting in a duplication process for two of the output branches. Note that two LLR inputs will be summed by each VND for the third output branch, which outputs the final a-posteriori LLR for the estimation of $\hat{x}_{a}, \hat{x}_{b}$ and $\hat{x}_{c}$.
} 
possible decoding processes, as shown in Fig. 4 and described as follows:

1) With Inter-Layer Feedback: When the bits $x_{a}$ of the BL are not successfully decoded, the iterative IL technique will be activated for exploiting the extrinsic information of BL $A$ fed back from the FEC decoder B. In this case, both the solid lines and the dashed lines shown in the decoder of Figs. 2 and 4 will be activated. More explicitly, the "VND4CND2-VND3" block of Fig. 4 will be utilized for extracting the extra LLR information $L_{e}\left(x_{a}^{i}\right)$ for BL $A$ based on both the extrinsic LLR $L_{e}\left(x_{b}^{i}\right)$ and the soft check information $y_{a b}$. Generally, the "VND4-CND2-VND3" block of Fig. 4 represents a process similar to that of the "VND3-CND2VND4" block of Fig. 4. After this stage, improved a-priori information is generated for the $\mathrm{BL} A$, which concludes the current IL decoding iteration. Afterwards, the receiver will return to the beginning of the flow chart shown in Fig. 4. The iterative IL decoding process continues, until the affordable number of iterations is exhausted or the BL $A$ is perfectly recovered, as shown in Fig. 4.

2) Without Inter-Layer Feedback: When the BL $A$ is successfully recovered, the layers $A$ and $B$ will be estimated by the hard decision block of Fig. 4. Afterwards, the receiver may discard layer $B$, depending on whether it is deemed to be error-free or not by the CRC check. In this case, only the solid lines of Figs. 2 and 4 will be activated.

Moreover, after decoding $\mathrm{BL} A$, the recovered error-free hard bits $x_{a}$ may be represented using infinite LLR values, indicating the hard bits $0 / 1$, respectively. Then, the CND 2 process invoked for generating the LLR $L\left(x_{b}^{i}\right)$ shown in Fig. 2 may be derived as follows using the boxplus operation

$$
\begin{aligned}
L\left(x_{b}^{i}\right) & =L\left(x_{a}^{i}\right) \boxplus L\left(x_{a b}^{i}\right) \\
& =\operatorname{sign}\left[L\left(x_{a}^{i}\right)\right] \cdot \operatorname{sign}\left[L\left(x_{a b}^{i}\right)\right] \cdot \min \left[\infty,\left|L\left(x_{a b}^{i}\right)\right|\right] \\
& +\log \left(1+e^{-\infty}\right)-\log \left(1+e^{-\infty}\right) \\
& =\operatorname{sign}\left(\tilde{x}_{a}^{i}\right) \cdot L\left(x_{a b}^{i}\right),
\end{aligned}
$$

where $\tilde{x}_{a}^{i}$ is the modulated version of the bit $x_{a}^{i}$ and the LLR input $L\left(x_{a b}^{i}\right)$ is obtained by soft demodulating the received signal $y_{a b}$.

Note that since the process of recovering $y_{b}$ from $y_{a b}$ expressed by Eq. (2) is essentially an LLR sign-flipping operation, it does not affect the absolute value of the LLR information of $x_{b}$. This implies that in this scenario our proposed IL technique is equivalent to the traditional UEP techniques, where layers $A$ and $B$ are encoded and decoded independently. Moreover, since BL $A$ is decoded independently without feedback from EL $B$, the two layers are only decoded once, without any extra complexity imposed on the receiver. Additionally, in practical applications, $\mathrm{BL} A$ may be reconstructed immediately when it is received, without waiting for the arrival of the EL $B$.

In both of the above cases, if the decoded bit sequence $\hat{x}_{a}$ of the BL is corrupted after the IL-FEC decoding stage of Fig. 2, it will be dropped together with the ELs $\hat{x}_{b}$ and $\hat{x}_{c}$. Otherwise they will all be forwarded to the H.264 decoder of Fig. 2 for reconstructing the video signal $\hat{s}$.

Note that in the above description, we have considered decoding layers $A$ and $B$ only. The decoding of layer $C$ is carried out in the same way but we have excluded it for the sake of simplifying our discussions.

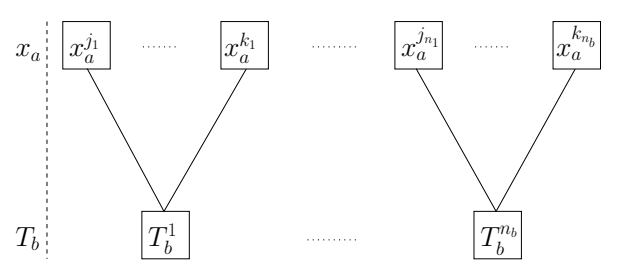

(a)
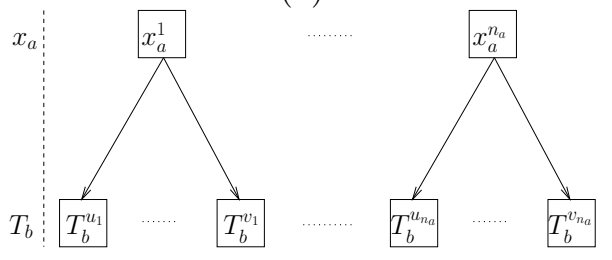

(b)

Fig. 5. Definition of $T_{b}^{1}, \cdots, T_{b}^{n_{b}}$ when the BL sequence $x_{a}$ and the EL sequence $x_{b}$ carry unequal length of bits.

\section{Inter-Layer FEC Coding for Layers Having Unequal Length}

In the above discussions, we assumed that the A, B and C partitions have an identical length. However, in practice they may carry an unequal number of bits. Here we detail the technique of applying our algorithm in the scenario, when the three partitions have an unequal length. Let us commence by assuming that the A, B, C partitions have the length of $n_{a}$, $n_{b}, n_{c}$ bits, respectively.

For the case of implanting $x_{a}$ into the systematic bits of $x_{b}$, the basic philosophy of the algorithm is to map/encode $x_{a}$ into a new bit sequence $t_{b}$, which has the same number of bits as the bitstream $x_{b}$ and will be implanted into the systematic bits of $x_{b}$ using the algorithm discussed in Section III-A. In other words, the bits $x_{a}$ will be replaced by the newly generated bits $t_{b}$ for the implantation process. Specifically, we introduce the sets $T_{b}^{1}, \cdots, T_{b}^{n_{b}}$ to assist in generating the stream $t_{b}$, where the relationship between $T_{b}^{1}, \cdots, T_{b}^{n_{b}}$ and the sequence $x_{b}$ is displayed in Fig. 5. For $n_{a}>n_{b}$, we split $x_{a}$ into $n_{b}$ number of groups on average as in Fig. $5(a)$, each constituting one of the sets $T_{b}^{1}, \cdots, T_{b}^{n_{b}}$. By contrast, for $n_{a}<n_{b}$, we split $T_{b}^{1}, \cdots, T_{b}^{n_{b}}$ into $n_{a}$ number of groups on average as in Fig. $5(b)$, where the sets $T_{b}^{1}, \cdots, T_{b}^{n_{b}}$ within the same group contain the same single bit of $x_{a}$. So far the sets $T_{b}^{1}, \cdots, T_{b}^{n_{b}}$ have been created from the bit sequence $x_{a}$. Then, each bit of the sequences $t_{b}$ will be generated from one of the sets $T_{b}^{1}, \cdots, T_{b}^{n_{b}}$ as follows:

$$
t_{b}^{i}=\sum_{x_{a}^{r} \in T_{b}^{i}} \oplus x_{a}^{r}, 0<i \leq n_{b} .
$$

Given the sequence $t_{b}$, we simply replace $x_{a}$ by $t_{b}$, when implanting the $x_{a}$ into the systematic bits of $x_{b}$. Therefore, $x_{a b}$ may be generated correspondingly using $x_{a b}^{i}=t_{b}^{i} \oplus x_{b}^{i}$. Similarly, the stream $x_{a}$ can be readily implanted into $x_{c}$ by introducing the bit sequence $t_{c}$ and the sets $T_{c}^{1}, \cdots, T_{c}^{n_{c}}$.

At the receiver, based on the technique detailed in Section III-B, decoder $\mathrm{A}$ is able to generate the extrinsic information of $x_{a}$. Decoder $\mathrm{B}$ is able to generate the extrinsic information of $t_{b}$ with the assistance of CND 2 of Fig. 2. Hence we design the technique to convert the extrinsic information between the sequence $x_{a}$ and $t_{b}$ for the sake of exchanging extrinsic information among the decoder A, CND 2 and decoder B of 
Fig. 2. Provided the LLR of $x_{a}$ and Eq. (3), the extrinsic LLR of $t_{b}$ may be readily derived using the boxplus operation as follows

$$
L_{e}\left(t_{b}^{i}\right)=L\left(\sum_{x_{a}^{r} \in T_{b}^{i}} \oplus x_{a}^{r}\right)=\sum_{x_{a}^{r} \in T_{b}^{i}} \boxplus L\left(x_{a}^{r}\right) .
$$

Similarly, provided the a-priori LLR of $x_{a}$ and the LLR of $t_{b}^{i}$, the extrinsic LLR of $x_{a}$ may be derived as follows.

1) When $n_{a}>n_{b}$, the extrinsic information of $x_{a}$ may be readily derived as

$$
\begin{aligned}
L_{e}\left(x_{a}^{i}\right) & =L\left(\sum_{x_{a}^{r} \in T_{b}^{i} \backslash x_{a}^{i}} \oplus x_{a}^{r} \oplus t_{b}^{i}\right) \\
& =\sum_{x_{a}^{r} \in T_{a}^{i} \backslash x_{a}^{i}} \boxplus L_{e}\left(x_{a}^{r}\right) \boxplus L\left(t_{b}^{i}\right) .
\end{aligned}
$$

2) When $n_{a}<n_{b}$, the extrinsic information of $x_{a}$ can be expressed as

$$
L_{e}\left(x_{a}^{i}\right)=\sum_{\forall T_{b}^{r}, x_{a}^{i} \in T_{b}^{r}} L_{e}^{r}\left(x_{a}^{r}\right) .
$$

Note that the basic idea of the above algorithm is to map the bits $x_{a}$ into a new bit sequence $t_{b}$, which is basically an encoder having a variable coding rate encoder. Hence, a number of codecs, such as low-density parity-check (LDPC) codes [42] and Luby transform (LT) [43] codes may be employed for the mapping of $x_{a}$ to the stream $t_{b}$. However, they may impose error-propagation in this specific scenario. Hence, in this treatise we employ the method detailed in this section to prevent error-propagation.

\section{IL-FEC Overheads}

The possible overheads imposed by our proposed technique are listed as follows:

1) Delay: Our technique is implemented using the partitioning mode of H.264, where each video frame may be encoded into a number of slices. These slices may be encoded into at most three partitions. Since the IL encoding and decoding process is performed within each slice, no extra delay is imposed by our proposed technique.

2) Complexity: As detailed in Section III-B, the signalflows are based on low-complexity operations compared to the FEC decoding. When the BL A can be recovered in its own right, only sign-flipping is necessitated for extracting the systematic LLR information of the ELs $B$ and $C$. Specifically, we impose a $21 \%$ extra complexity ${ }^{5}$, as it will be detailed in Section V-C.

3) FEC-redundancy: The BL $A$ does not rely on the ELs for its decoding operations and the systematic LLR information of the ELs $B$ and $C$ can be extracted from the received check information $y_{a b}$ and $y_{a c}$ without any loss, provided that the BL is perfectly decoded. Furthermore, since the transmitted bit sequences $x_{a b}$ and $x_{a c}$ have the same length as that of the bit sequence $x_{b}$ and $x_{c}$, respectively, we do not impose any extra

\footnotetext{
${ }^{5}$ According to our experiments, it is sufficient to use a single iteration, which results in a low complexity.
}

protection bits. Hence the IL-FEC does not impose extra FEC redundancy.

\section{Mutual Information AnAlysis}

\begin{tabular}{|l|r||l|r|}
\hline System Parameters & Value & System Parameters & Value \\
\hline \hline FEC & RSC $[1011,1101,1111]$ & Number of Tx antennas & 4 \\
\hline Modulation & QPSK & Elements Per AA & 4 \\
\hline Channel & Narrowband Rayleigh & Number of Rx antennas & 4 \\
\cline { 3 - 4 } & Fading Channel & Overall Coding Rate & $1 / 2$ \\
\hline
\end{tabular}

TABLE I

PARAMETERS EMPLOYED IN OUR SYSTEMS, WHERE “AA” INDICATES ANTENNA ARRAY.

\begin{tabular}{|l|r|r|r|}
\hline Error Protection & \multicolumn{3}{|c|}{ Code Rates } \\
\cline { 2 - 4 } Arrangements & $L_{0}$ & $L_{1}$ & Average \\
\hline EEP & 0.5 & 0.5 & 0.5 \\
\hline UEP1 & 0.54 & 0.46 & 0.5 \\
\hline UEP2 & 0.47 & 0.53 & 0.5 \\
\hline
\end{tabular}

TABLE II

CODING RATES OF RSC CODEC ERROR PROTECTION ARRANGEMENTS FOR THE BL $L_{0}$ AND THE EL $L_{1}$. THE CODE-RATES WERE ADJUSTED BY VARIABLE-RATE PUNCTURERS.

In this section, we analyze our proposed system using $\mathrm{MI}^{6}$. For the sake of simplifying the analysis, we assume that there are two layers: a BL $L_{0}$ and an EL $L_{1}$. Furthermore, we employed a $1 / 3$ RSC having the generator polynomials $[1011,1101,1111]^{7}$. The system parameters used in our simulations are summarized in Table IV. In the following analysis, where two layers are considered, the BL is protected by the ILFEC codec. Hence, we consider the convergence behavior of the BL. For the sake of analyzing our IL-FEC codec, different error protection arrangements were considered, as shown in Table II.

In Fig. 6, we plot the extrinsic MI at the output of the RSC decoder for different $E_{b} / N_{0}$ values for all the codes in Table II. Observe from Fig. 6 that the schemes employing our iterative inter-layer technique always acquire a higher MI value than those dispensing with the IL-FEC technique. For example, the RSC-EEP scheme and RSC-EEP-IL scheme generate 0.91 and $0.975^{8}$ extrinsic information at $-8 \mathrm{~dB}$. This improvement is attained by our proposed scheme due to the fact that extra MI is fed back to the BL from the EL.

\section{System Performance}

Let us continue by benchmarking our proposed IL-FECLSSTC system against the traditional UEP aided FEC-LSSTC system using a RSC. Two 30-frame video sequences, namely the Foreman and Football clips, represented in $(352 \times 288)$ pixel common intermediate format (CIF) and 4:2:0 YUV format were encoded using the JM/AVC $15.1 \mathrm{H} .264$ reference

\footnotetext{
${ }^{6} \mathrm{MI}$ is known as a metric to represent the confidence of a signal sequence. Generally bigger MI indicates lower BER value of the measured signal sequence, while lower BER normally indicates lower PLR.

${ }^{7}$ The first polynomial indicates the feedback parameter, while the other two polynomials represent the feed-forward parameters. The code rates were adjusted by variable-rate puncturers.

${ }^{8}$ Larger amount of extrinsic information indicates a lower BER [44].
} 


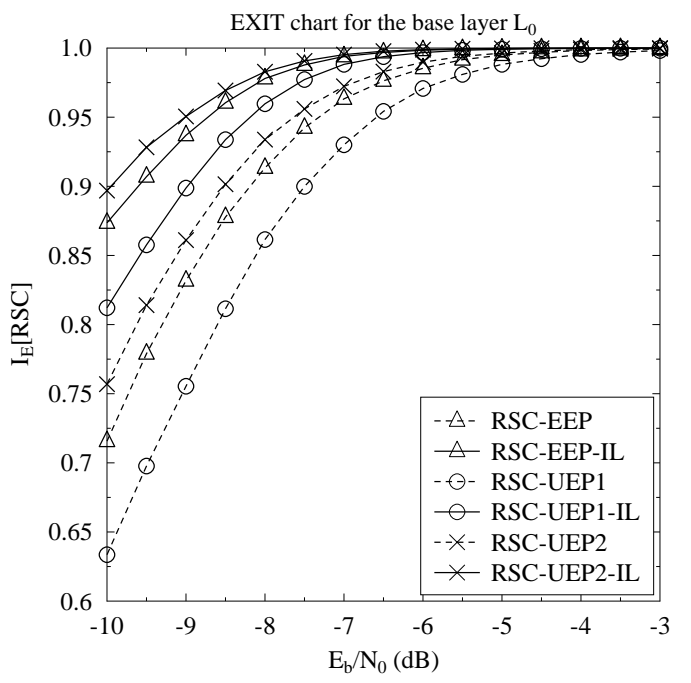

Fig. 6. Extrinsic information generated by the RSC decoders for all error protection arrangements of Table II.

\begin{tabular}{|l|r|r|}
\hline & Football & Foreman \\
\hline \hline Representation & YUV 4:2:0 & YUV 4:2:0 \\
\hline Format & CIF & CIF \\
\hline Bits Per Pixel & 8 & 8 \\
\hline FPS & 15 & 30 \\
\hline Number of Frames & 30 & 30 \\
\hline Video Codec & H.264 PM & H.264 PM \\
\hline Bitrate & $1522 \mathrm{kbps}$ & $655 \mathrm{kbps}$ \\
\hline Error-Free PSNR & $37.6 \mathrm{~dB}$ & $38.4 \mathrm{~dB}$ \\
\hline Error Concealment & Motion-Copy & Motion-Copy \\
\hline
\end{tabular}

TABLE III

THE PARAMETERS OF THE VIDEO SEQUENCES EMPLOYED

video codec operated in its data partitioning aided mode. The video scanning rates expressed in frame per second (FPS) were 30 and 15 for the Foreman and Football sequences, respectively. The motion-copy ${ }^{9}$, based error concealment tool built into the H.264 reference codec was employed for the sake of combating the effects of channel impairments. Moreover, the H.264 encoder was configured to generate fixed-byte ${ }^{10}$ slices, as defined in [4]. Both of the 30-frame video sequences were encoded into an intra-coded (I) frame, followed by 29 predicted $(\mathrm{P})$ frames. The bi-directionally predicted (B) frame was disabled due to the fact that it relies on both previous and future frames for decoding, which may introduce more error propagation as well as additional delay. All the above configurations jointly result in a bitrate of $655 \mathrm{kbps}$ and an error-free peak-signal to noise ratio (PSNR) of $38.4 \mathrm{~dB}$ for the Foreman sequence. On the other hand, the coded Football bitstream has a bitrate of $1522 \mathrm{kbps}$ and an error-free PSNR of $37.6 \mathrm{~dB}$. We employed the Foreman and Football sequences in order to show the suitability of our scheme for the transmission of both low-motion and high-motion video. The parameters of the video sequences employed are shown in Table $\mathrm{V}$, while our system parametes are listed in Table IV.

\footnotetext{
${ }^{9}$ When the information of a macroblock (MB) is lost, the motion vector of this MB may be copied or estimated from its adjacent MBs or previously decoded reference frames. Then, the MB may be reconstructed using the estimated motion vector.

${ }^{10}$ In this mode, the H.264/AVC codec will endeavor to encode a frame into multiple slices, each having a fixed number of bytes.
}

The H.264-compressed bitstream was FEC encoded and transmitted on a network abstract layer unit (NALU) [4] basis, which is the smallest element to be used by the source decoder. At the receiver, each error-infested NALU must be dropped by the video decoder, if errors are detected by the CRC check. All experiments were repeated 100 times for the sake of generating smooth performance curves.

Below, we will firstly describe the error-protection arrangements in Section V-A. Then we will characterize the attainable BER versus channel SNR performance and PSNR versus channel SNR performance employing a lower-complexity RSC codec in Section V-B. Finally, in Section V-C we will quantify the system's computational complexity by counting the number of decoding operations executed.

\section{A. Error Protection Arrangements}

\begin{tabular}{|l|r|r|r|r|}
\hline Error Protection & \multicolumn{4}{|c|}{ Code Rates } \\
\cline { 2 - 5 } Arrangements & Type A & Type B & Type C & Average \\
\hline EEP & $0.5 / 0.5$ & $0.5 / 0.5$ & $0.5 / 0.5$ & $0.5 / 0.5$ \\
\hline UEP1 & $0.35 / 0.40$ & $0.57 / 0.65$ & $0.57 / 0.65$ & $0.5 / 0.5$ \\
\hline UEP2 & $0.45 / 0.55$ & $0.52 / 0.46$ & $0.52 / 0.46$ & $0.5 / 0.5$ \\
\hline UEP3 & $0.65 / 0.60$ & $0.47 / 0.43$ & $0.47 / 0.43$ & $0.5 / 0.5$ \\
\hline UEP4 & $0.75 / 0.70$ & $0.45 / 0.39$ & $0.45 / 0.39$ & $0.5 / 0.5$ \\
\hline UEP5 & $0.85 / 0.80$ & $0.44 / 0.37$ & $0.44 / 0.37$ & $0.5 / 0.5$ \\
\hline UEP6 & $0.95 / 0.90$ & $0.43 / 0.35$ & $0.43 / 0.35$ & $0.5 / 0.5$ \\
\hline
\end{tabular}

TABLE IV

CODING RATES OF DIFFERENT ERROR PROTECTION ARRANGEMENTS FOR THE FOOTBALl/FOREMAN SEQUENCE. THE CODE-RATES WERE

ADJUSTED BY VARIABLE-RATE PUNCTURERS.

In the simulations, we employ the overall coding rate ${ }^{11}$ of $1 / 2$ for both EEP and UEP schemes. For each compressed bitstream, all NALUs were scanned to calculate the total number of bits for the $\mathrm{A}, \mathrm{B}$, and $\mathrm{C}$ partitions. Let us assume that the A, B and $\mathrm{C}$ partitions have a total $N_{a}, N_{b}$ and $N_{c}$ bits, respectively. The $\mathrm{A}, \mathrm{B}, \mathrm{C}$ streams have coding rates of $r_{a}, r_{b}$ and $r_{c}$, respectively. Then the following equation must be satisfied for the sake of guaranteeing that the overall coding rate remains $1 / 2$ :

$$
2 \times\left(N_{a}+N_{b}+N_{c}\right)=\frac{N_{a}}{r_{a}}+\frac{N_{b}}{r_{b}}+\frac{N_{c}}{r_{c}} .
$$

Again, the A stream is the most important layer, while the $\mathrm{B}$ and type $\mathrm{C}$ bitstreams are the ELs, where the bitstream B and $\mathrm{C}$ are similarly important. Hence in all the error protection arrangements we have $r_{b}=r_{c}$. More specifically, we first select a specific value to $r_{a}$, then the value of $r_{b}=r_{c}$ was calculated as follows:

$$
r_{b}=\frac{N_{b}+N_{c}}{2 \times\left(N_{a}+N_{b}+N_{c}\right)-\frac{N_{a}}{r_{a}}} .
$$

Note that the total number of bits for each partitions of the different video sequences may be different, which results in different protection arrangements. Based on the above, the five error protection arrangements conceived for the Football and Foreman sequences are shown in Table IV, which may be readily combined with arbitrary EEP or UEP schemes, where variable-rate puncturers were designed and employed to achieve a specific coding rate.

\footnotetext{
${ }^{11}$ Arbitrary overall coding rates such as $2 / 3,1 / 3,1 / 4$, etc. can be readily applied by changing the channel codec parameters and the puncturers.
} 


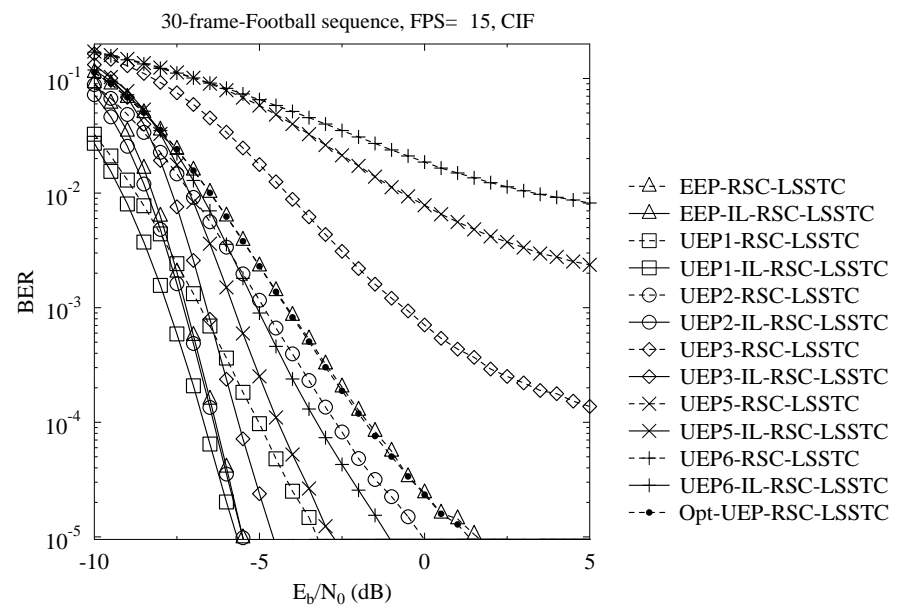

Fig. 7. BER versus $E_{b} / N_{0}$ performance for the A partition of the Football sequence, including the RSC coding schemes of Table IV and the Opt-UEPRSC-LSSTC [20].

\section{B. System Performance using RSC Codec}

In this section, we benchmark our proposed system using the RSC codec of Table IV. All the error protection arrangements of Section V-A will be utilized. Furthermore, in [20] an UEP algorithm was proposed, which the authors of [20] referred to as the optimal UEP. We used this scheme as a benchmarker, which we refer to as the Opt-UEP-RSC-LSSTC arrangement.

The BER curves of the A partition in the Football sequence are displayed in Fig. 7, where the performance of the error protection schemes of Table IV are illustrated. Observe in Fig. 7 that the schemes using the IL-RSC codec achieve a reduced BER compared to their benchmarkers. Specifically, the EEPIL-RSC-LSSTC scheme outperforms the EEP-RSC-LSSTC benchmarker by about $7.2 \mathrm{~dB}$ at a BER of $10^{-5}$. Furthermore, among all the error protection arrangements, the UEP1-ILRSC-LSSTC scheme achieves the best BER performance due to the strong error protection assigned for the A partition. Hence, we may conclude that the UEP aided IL-RSC schemes are capable of providing an improved system performance compared to the traditional UEP aided RSC codec. On the other hand, the Opt-UEP-RSC-LSSTC system achieves similar BER performance to that of the EEP-RSC-LSSTC scheme.

The BER versus $E_{b} / N_{0}$ performance of the B partition for the Football sequence is presented in Fig. 8. Similar trends were observed for the $\mathrm{C}$ partition as well, which are not included here owing to space-economy. Observe in Fig. 8 that the performance of the schemes using IL-RSC is slightly worse than that of their benchmarkers. This is due to the fact that more errors may be introduced into the $\mathrm{B}$ partition, when the A partition cannot be correctly decoded. In this scenario the $\mathrm{B}$ partition must be dropped in the traditional UEP aided RSC-LSSTC schemes. Hence the error propagation to the B partition does not further degrade the situation.

The PSNR versus $E_{b} / N_{0}$ performance recorded for the Football sequence is shown in Fig. 9, where we observe that the EEP-RSC-LSSTC scheme achieves the best performance among all the systems without IL techniques, because the A partition carries only the video header information and fails to assist the H.264 decoder in concealing the residual

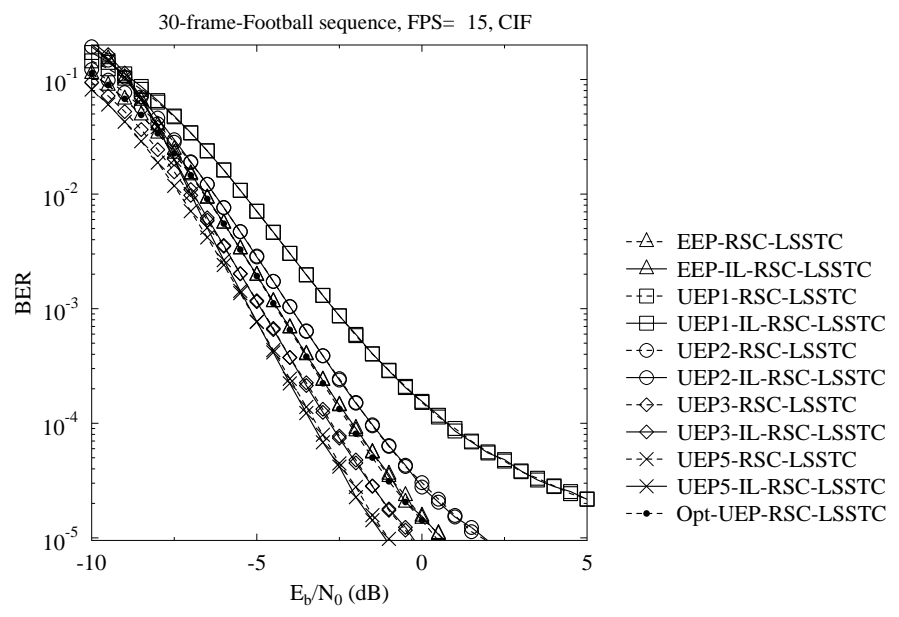

Fig. 8. BER versus $E_{b} / N_{0}$ performance for the B partition of the Football sequence, including the RSC coding schemes of Table IV and the Opt-UEPRSC-LSSTC [20].

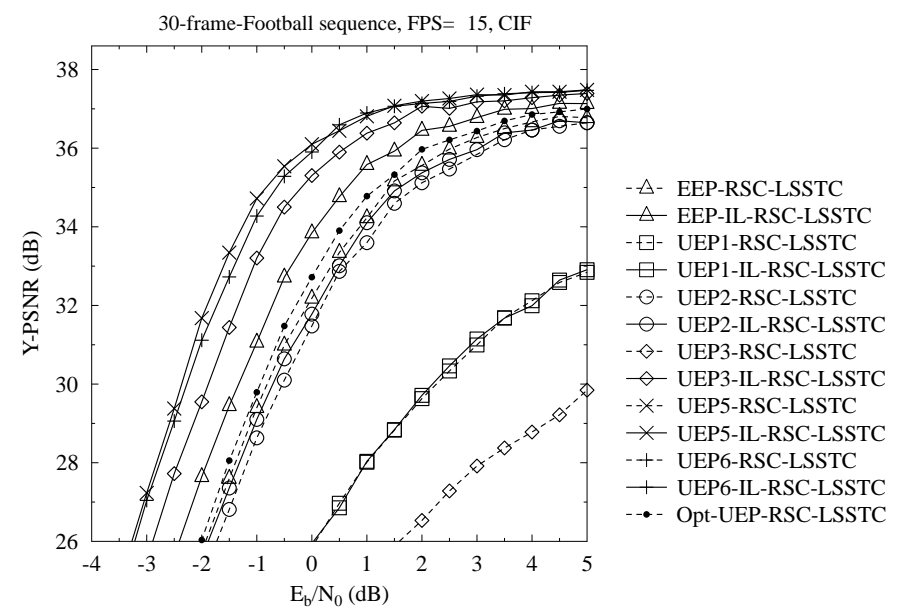

Fig. 9. PSNR versus $E_{b} / N_{0}$ performance for the Football sequence, including the RSC coding schemes of Table IV and the Opt-UEP-RSC-LSSTC [20].

errors, when the $\mathrm{B}$ and $\mathrm{C}$ partitions are corrupted. Furthermore, the systems using our proposed IL-RSC-LSSTC model outperform their corresponding benchmarkers. Specifically, the UEP5-IL-RSC-LSSTC constitutes the best protection arrangement among all IL-RSC schemes, which achieves a power reduction of about $3 \mathrm{~dB}^{12}$ compared to the EEP-RSC-LSSTC scheme at a PSNR of $36 \mathrm{~dB}$. Alternatively, about $3.7 \mathrm{~dB}$ of PSNR video quality improvement may be observed at a channel SNR of $0 \mathrm{~dB}$. On the other hand, the Opt-UEPRSC-LSSTC system dispensing with the IL technique slightly outperforms the EEP-RSC-LSSTC scheme, namely by a power reduction of about $0.5 \mathrm{~dB}$ at a PSNR of $36 \mathrm{~dB}$. The UEP5IL-RSC-LSSTC substantially outperforms the Opt-UEP-RSCLSSTC arrangement, namely by a power reduction of about $2.5 \mathrm{~dB}$ at a PSNR of $36 \mathrm{~dB}$ or alternatively, about $3.4 \mathrm{~dB}$ of PSNR video quality improvement may be observed at an $E_{b} / N_{0}$ of $0 \mathrm{~dB}$. A subjective comparison of the UEP5IL-RSC-LSSTC and EEP-RSC-LSSTC arrangements for the Football sequence is presented in Fig. 11.

\footnotetext{
${ }^{12}$ The power reduction is read horizontally. Specifically, the UEP5-IL-RSCLSSTC achieves the PSNR of $36 \mathrm{~dB}$ with $3 \mathrm{~dB}$ less power than the EEPRSC-LSSTC scheme.
} 


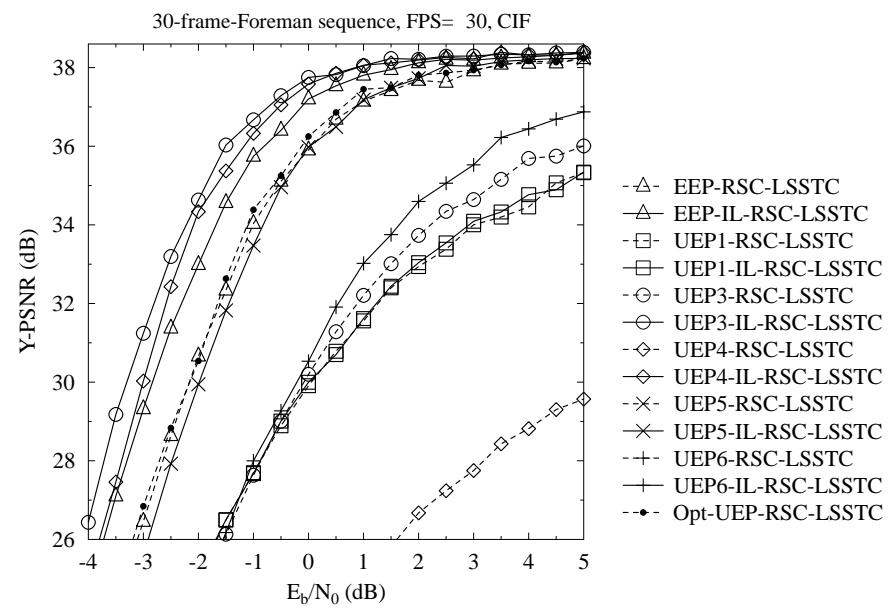

Fig. 10. PSNR versus $E_{b} / N_{0}$ performance for the Foreman sequence, including the RSC coding schemes of Table IV and the Opt-UEP-RSC-LSSTC [20].

For providing further insights for video scenes having different motion-activity, the PSNR versus $E_{b} / N_{0}$ performance of the IL-RSC-LSSTC model is presented in Fig. 10 using the Foreman sequence, when employing the protection arrangements of Table IV. Similar to the Football sequence, the traditional UEP technique can hardly improve the reconstructed video quality by allocating more FEC redundancy to the more important layers. By contrast, about $2 \mathrm{~dB}$ of power reduction is achieved by the UEP3-IL-RSC-LSSTC arrangement compared to the EEP-RSC-LSSTC scheme at a PSNR of $37 \mathrm{~dB}$. Alternatively, about $3.2 \mathrm{~dB}$ of PSNR video quality improvement may be observed at a channel SNR of $-1 \mathrm{~dB}$. Similar to the Football sequence, a limited gain can be observed for the Opt-UEP-RSC-LSSTC system compared to the EEP-RSC-LSSTC scheme, while the UEP5IL-RSC-LSSTC substantially outperforms the Opt-UEP-RSCLSSTC, namely by about $1.8 \mathrm{~dB}$ at a PSNR of $37 \mathrm{~dB}$. A subjective comparison of the UEP3-IL-RSC-LSSTC and EEP-RSC-LSSTC arrangements for the Foreman sequence is presented in Fig. 11.

We may conclude from the above discussion that the A partition should be assigned a code-rate of 0.85 and 0.60 for the Football and Foreman sequence, respectively, for the sake of achieving the best overall system performance, when employing the RSC codec, which contradicts to the traditional UEP strategy. The main reason for this is that the inter-layer aided RSC decoder can still successfully recover the weaker protected A partition relying on the extrinsic information fed back from the $\mathrm{B}$ and $\mathrm{C}$ partitions with the aid of inter-layer decoding, because $\mathrm{B}$ and $\mathrm{C}$ are more strongly protected than the A partition.

\section{Complexity Analysis}

In order to provide insights into the complexity of our scheme, we benchmark the complexity of our IL-FEC-LSSTC scheme using both the RSC codec in Fig. 12. We emphasize that if the A partition was corrupted, the corresponding complexity imposed by the $\mathrm{B}$ and $\mathrm{C}$ partitions was not taken into account, since they cannot be utilized by the video decoder in this case. Therefore, the complexity of both the IL-FEC-
LSSTC system and of the benchmarkers is directly proportional to the $E_{b} / N_{0}$ value. Furthermore, in the simulations each NALU was encoded by the FEC as a single packet. The total computational complexity is dominated by that of FEC decoding. Hence, the total number of FEC decoding operations substantially affects the system's complexity, which was hence used for comparing the system's complexity. The y-axis of Fig. 12 represents the average number of RSC decoding operations per NALU, which was averaged over 2221 NALUs in the H.264 encoded Football bitstream for the sake of statistical relevance, where again each NALU was encoded as a single packet in the experiments.

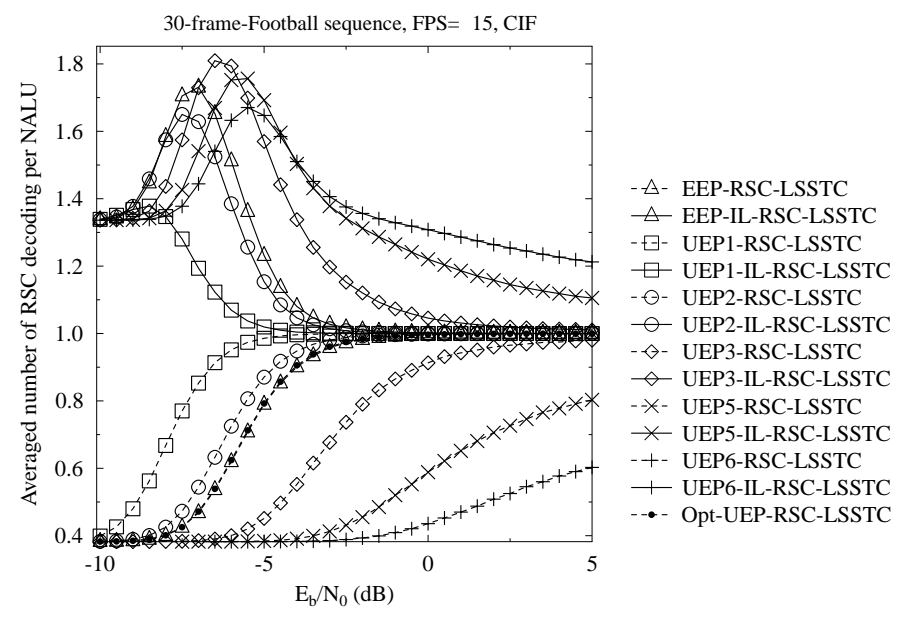

Fig. 12. Complexity comparison of the Opt-UEP-RSC-LSSTC system, the IL-RSC-LSSTC schemes and the classic RSC-LSSTC schemes for the error protection arrangements of Table IV for the Football sequence.

Observe from Fig. 12 that each curve of the IL-RSCLSSTC schemes may be divided into two regions, where the complexity of the systems increases and decreases upon the increasing $E_{b} / N_{0}$. For example, the curve of the UEP3-ILRSC-LSSTC scheme can be split at $E_{b} / N_{0}$ of about -6.5 dB. Specifically, in the $E_{b} / N_{0}$ region of $[-10,-6.5] \mathrm{dB}$, the complexity of the UEP3-IL-RSC-LSSTC scheme increases upon increasing the $E_{b} / N_{0}$ value. This is due to the fact that the IL decoding technique was activated frequently for assisting the decoding of A partition. By contrast, for higher $E_{b} / N_{0}$ values the A partition is more likely to be recovered with the aid of the IL technique, which in turn results in decoding the $\mathrm{B}$ and $\mathrm{C}$ partitions more than once. In the $E_{b} / N_{0}$ region of $[-6.5,5] \mathrm{dB}$, the complexity of the UEP3-ILRSC-LSSTC scheme decreases upon increasing $E_{b} / N_{0}$ value. The reason for this phenomenon is that the IL decoding technique is less frequently activated, when the A partition is more likely to be perfectly decoded in its own right at higher $E_{b} / N_{0}$ values. Moreover, the complexity of all the RSC-LSSTC schemes increases upon increasing $E_{b} / N_{0}$. This may be attributed to the fact that at lower $E_{b} / N_{0}$ the B and $\mathrm{C}$ partition were more likely to be dropped by the decoder due to the corruption of the A partition. Since low $E_{b} / N_{0}$ results in unacceptable video quality, here we only focus on higher $E_{b} / N_{0}$ region. More specifically, the UEP5-IL-RSCLSSTC scheme achieves $E_{b} / N_{0}$ gains of $3 \mathrm{~dB}$ and $2.5 \mathrm{~dB}$ by imposing about $21 \%$ higher complexity than the EEPRSC-LSSTC and Opt-UEP-RSC-LSSTC schemes at a video 


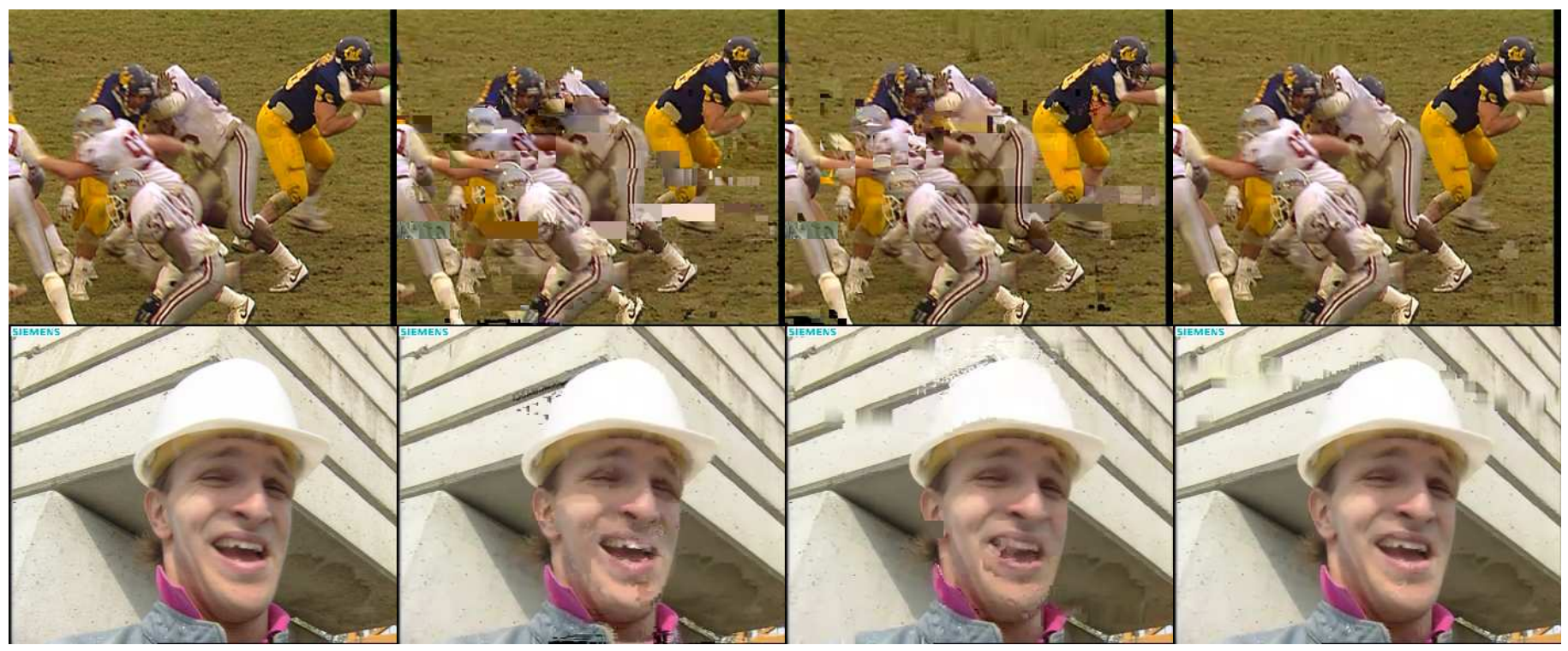

Fig. 11. Video comparison at $E_{b} / N_{0}=-2.5 \mathrm{~dB}$ for the Football and Foreman sequences. The first column indicates the original frames. The second column indicates the EEP-IL-RSC-LSSTC decoded frames. The third column indicates the Opt-UEP-RSC-LSSTC [20] decoded frames. The fourth column represents the UEP5-IL-RSC-LSSTC and UEP3-IL-RSC-LSSTC decoded frames for the Football and Foreman sequences, respectively.

quality of $36 \mathrm{~dB}$, respectively. Alternatively, the UEP5-ILRSC-LSSTC has PSNR gains of $3.7 \mathrm{~dB}$ and $3.4 \mathrm{~dB}$ at the cost of a $21 \%$ complexity increase compared to the EEP-RSCLSSTC and Opt-UEP-RSC-LSSTC schemes at an $E_{b} / N_{0}$ of $0 \mathrm{~dB}$, respectively.

In conclusion of the Section V:

1) In the RSC based systems, the most important layer should be assigned less redundancy than partitions $\mathrm{B}$ and $\mathrm{C}$ for the sake of achieving the best overall system performance, which is in contrast to the traditional UEP strategy. For example, the system arrangement having channel coding rates of $0.85,0.44$ and 0.44 for the $\mathrm{A}, \mathrm{B}$ and $\mathrm{C}$ partitions, respectively, achieves the best system performance when employing the RSC code for the transmission of the Football sequence.

2) As jointly observed from Fig. 9 of Section V-B and Fig. 12 of $\mathrm{V}-\mathrm{C}$, our proposed IL coding technique is capable of achieving $2.5 \mathrm{~dB}$ of $E_{b} / N_{0}$ again or alternatively, 3.4 $\mathrm{dB}$ of PSNR gain over the traditional UEP technique at the cost of a $21 \%$ complexity increase.

\section{CONCLUSIONS}

An IL-FEC coded video scheme relying on multi-functional MIMOs was proposed for mobile TV broadcasting, where the data partitioning mode of H.264 video coding was utilized and the systematic bits of the A partition were incorporated into the systematic bits of the $\mathrm{B}$ and $\mathrm{C}$ partitions using an XOR operation. At the receiver, our IL-FEC decoding technique of Fig. 2 was activated for the sake of attaining an improved system performance. A RSC codec were invoked for demonstrating that the proposed scheme is capable of substantially outperforming the traditional UEP FEC codecs. The system advocated was analyzed using mutual information for providing insights into the gain attained using our IL-FEC coding scheme.

In our future work, we will incorporate the IL-FEC scheme into SVC and multiview video coding. Moreover, we will also carry out further investigations for optimizing the inter-layer coded system performance.

\section{REFERENCES}

[1] T. Zhang and Y. Xu, "Unequal packet loss protection for layered video transmission," IEEE Transactions on Broadcasting, vol. 45, pp. 243 252, June 1999.

[2] H. Imaizumi and A. Luthra, Three-Dimensional Television, Video, and Display Technologies, ch. MPEG-2 Multiview Profile, pp. 169-181. Berlin, Heidelberg, and New York: Springer Verlag, 2002.

[3] H. Schwarz, D. Marpe, and T. Wiegand, "Overview of the scalable video coding extension of the H.264/AVC standard," IEEE Transactions on Circuits and Systems for Video Technology, vol. 17, pp. 1103-1120, September 2007

[4] Joint Video Team (JVT) of ISO/IEC MPEG and ITU-T VCEG, ITU$T$ Rec. H.264/ISO/IEC 14496-10 AVC: Advanced Video Coding for Generic Audiovisual Services, March 2010.

[5] A. Vetro, T. Wiegand, and G. Sullivan, "Overview of the stereo and multiview video coding extensions of the H.264/MPEG-4 AVC standard,' Proceedings of the IEEE, vol. 99, pp. 626-642, April 2011.

[6] L. Hanzo, P. Cherriman, and J. Streit, Video Compression and Communications: From Basics to H.261, H.263, H.264, MPEG2, MPEG4 for DVB and HSDPA-Style Adaptive Turbo-Transceivers. New York: John Wiley, 2007.

[7] F. Yang, Q. Zhang, W. Zhu, and Y.-Q. Zhang, "End-to-end TCP-friendly streaming protocol and bit allocation for scalable video over wireless Internet," IEEE Journal on Selected Areas in Communications, vol. 22 pp. 777-790, May 2004.

[8] B. Masnick and J. Wolf, "On linear unequal error protection codes," IEEE Transactions on Information Theory, vol. 13, pp. 600-607, October 1967.

[9] T. Brüggen and P. Vary, "Unequal error protection by modulation with unequal power allocation," IEEE Communications Letters, vol. 9, pp. 484-486, June 2005

[10] V. Pavlushkov, R. Johannesson, and V. Zyablov, "Unequal error protection for convolutional codes," IEEE Transactions on Information Theory, vol. 52, pp. 700-708, February 2006.

[11] J. Hagenauer, "Rate-compatible puncture convolutional codes (RCPC) and their application," IEEE Transactions on Communications, vol. 36, pp. 389-400, April 1988.

[12] N. Rahnavard and F. Fekri, "New results on unequal error protection using LDPC codes," IEEE Communications Letters, vol. 10, pp. 43-45, January 2006.

[13] V. Kumar and O. Milenkovic, "On unequal error protection LDPC codes based on Plotkin-type constructions," IEEE Transactions on Communications, vol. 54, pp. 994-1005, June 2006

[14] C. Gong, G. Yue, and X. Wang, "Message-wise unequal error protection based on low-density parity-check codes," IEEE Transactions on Communications, vol. 59, pp. 1019-1030, April 2011. 
[15] N. Rahnavard, H. Pishro-Nik, and F. Fekri, "Unequal error protection using partially regular LDPC codes," IEEE Transactions on Communications, vol. 55, pp. 387-391, March 2007.

[16] H. Wang, F. Zhai, Y. Eisenberg, and A. Katsaggelos, "Cost-distortion optimized unequal error protection for object-based video communications," IEEE Transactions on Circuits and Systems for Video Technology, vol. 15 , pp. 1505-1516, December 2005

[17] S. Ng, J. Chung, and L. Hanzo, "Turbo-detected unequal protection MPEG-4 wireless video telephony using multi-level coding, trellis coded modulation and space-time trellis coding," IEE Proceedings Communications, vol. 152, pp. 1116-1124, December 2005.

[18] Nasruminallah, M. El-Hajjar, N. Othman, A. Quang, and L. Hanzo, "Over-complete mapping aided, soft-bit assisted iterative unequal error protection H.264 joint source and channel decoding," IEEE 68th Vehicular Technology Conference, VTC 2008-Fall, pp. 1-5, September 2008.

[19] M. Aydinlik and M. Salehi, "Turbo coded modulation for unequal error protection," IEEE Transactions on Communications, vol. 56, pp. 555564, April 2008

[20] H. Ha and C. Yim, "Layer-weighted unequal error protection for scalable video coding extension of H.264/AVC," IEEE Transactions on Consumer Electronics, vol. 54, pp. 736-744, May 2008.

[21] Y. Chang, S. Lee, and R. Komyia, "A fast forward error correction allocation algorithm for unequal error protection of video transmission over wireless channels," IEEE Transactions on Consumer Electronics, vol. 54, pp. 1066-1073, August 2008.

[22] Y. C. Chang, S. W. Lee, and R. Komiya, "A low complexity hierarchical QAM symbol bits allocation algorithm for unequal error protection of wireless video transmission," IEEE Transactions on Consumer Electronics, vol. 55, pp. 1089-1097, August 2009.

[23] E. Maani and A. Katsaggelos, "Unequal error protection for robust streaming of scalable video over packet lossy networks," IEEE Transactions on Circuits and Systems for Video Technology, vol. 20, pp. 407416, March 2010.

[24] S. Ahmad, R. Hamzaoui, and M. Al-Akaidi, "Unequal error protection using fountain codes with applications to video communication," IEEE Transactions on Multimedia, vol. 13, pp. 92-101, February 2011.

[25] C. Hellge, T. Schierl, and T. Wiegand, "Multidimensional layered forward error correction using rateless codes," in IEEE International Conference on Communications, 2008, pp. 480-484, May 2008.

[26] C. Hellge, D. Gomez-Barquero, T. Schierl, and T. Wiegand, "Layeraware forward error correction for mobile broadcast of layered media," IEEE Transactions on Multimedia, vol. 13, pp. 551-562, June 2011.

[27] Y. Huo, X. Zuo, R. G. Maunder, and L. Hanzo, "Interlayer FEC decoded multi-layer video streaming," IEEE Global Telecommunications Conference, 2012, to appear. Available at http: / / eprints.soton.ac.uk/343750/1/1569586913.pdf.

[28] M. El-Hajjar and L. Hanzo, "Layered steered space-time codes and their capacity," Electronics Letters, vol. 43, pp. 680-682, June 2007.

[29] L. Hanzo, O. Alamri, M. El-Hajjar, and N. Wu, Near-Capacity MultiFunctional MIMO Systems: Sphere-Packing, Iterative Detection and Cooperation. John Wiley \& Sons, IEEE press, 2009.

[30] P. Wolniansky, G. Foschini, G. Golden, and R. Valenzuela, "V-BLAST: an architecture for realizing very high data rates over the rich-scattering wireless channel," in International Symposium on Signals, Systems, and Electronics, ISSSE, pp. 295-300, September 1998.

[31] V. Tarokh, H. Jafarkhani, and A. Calderbank, "Space-time block codes from orthogonal designs," IEEE Transactions on Information Theory, vol. 45, pp. 1456-1467, July 1999

[32] J. S. Blogh and L. Hanzo, Third-Generation Systems and Intelligent Wireless Networking: Smart Antennas and Adaptive Modulation. New York, NY, USA: Halsted Press, 2002.

[33] S. ten Brink, "Convergence behavior of iteratively decoded parallel concatenated codes," IEEE Transactions on Communications, vol. 49 pp. 1727-1737, October 2001.

[34] Y. Huo, M. EI-Hajjar, M. F. U. Butt, and L. Hanzo, "Inter-layer-decoding aided self-concatenated coded scalable video transmission," IEEE Wireless Communications and Networking Conference, WCNC 2013, accepted. Available at http: / / eprints.soton.ac.uk/346610/2/IL_SECCC.pdf.

35] Nasruminallah and L. Hanzo, "EXIT-chart optimized short block codes for iterative joint source and channel decoding in H.264 video telephony," IEEE Transactions on Vehicular Technology, vol. 58, pp. 43064315, October 2009.

[36] Nasruminallah and L. Hanzo, "Near-capacity H.264 multimedia communications using iterative joint source-channel decoding," IEEE Communications Surveys and Tutorials, vol. 14, pp. 538-564, Second Quarter 2012.

[37] S. Wenger, "H.264/AVC over IP," IEEE Transactions on Circuits and Systems for Video Technology, vol. 13, pp. 645-656, July 2003.
[38] S. ten Brink, G. Kramer, and A. Ashikhmin, "Design of low-density parity-check codes for modulation and detection," IEEE Transactions on Communications, vol. 52, pp. 670-678, April 2004

[39] J. Hagenauer, E. Offer, and L. Papke, "Iterative decoding of binary block and convolutional codes," IEEE Transactions on Information Theory, vol. 42, pp. 429-445, March 1996.

[40] J. Chen, A. Dholakia, E. Eleftheriou, M. Fossorier, and X.-Y. Hu, "Reduced-complexity decoding of LDPC codes," IEEE Transactions on Communications, vol. 53, pp. 1288-1299, August 2005.

[41] C. Berrou, A. Glavieux, and P. Thitimajshima, "Near Shannon limit error-correcting coding and decoding: Turbo codes," in Proceedings of the International Conference on Communications, (Geneva, Switzerland), pp. 1064-1070, May 1993.

[42] R. Gallager, "Low-density parity-check codes," IEEE Transactions on Information Theory, pp. 21-28, 1962.

[43] M. Luby, "LT codes," in Proc. 43rd Annual IEEE Symposium Foundations Computer Science (FOCS), pp. 271-280, 2002.

[44] R. Otnes and M. Tüchler, "EXIT chart analysis applied to adaptive turbo equalization," in in Proceedings of Nordic Signal Processing Symposium, 2002

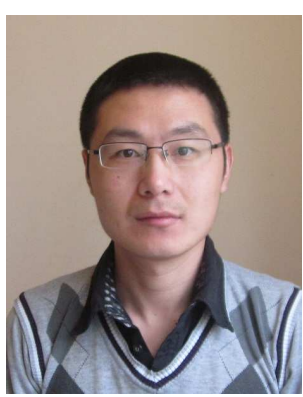

Yongkai Huo received the B.Eng. degree with distinction in computer science and technology from Hefei University of Technology, Hefei, China, in 2006 and the M.Eng. degree in computer software and theory from University of Science and Technology of China, Hefei, China, in 2009. He is currently working toward the Ph.D. degree with the Communications, Signal Processing and Control Group, School of Electronics and Computer Science, University of Southampton, Southampton, U.K. He received a scholarship under the China-U.K. Scholarships for Excellence Programme. His research interests include distributed video coding, multiview video coding, robust wireless video streaming and joint source-channel decoding.

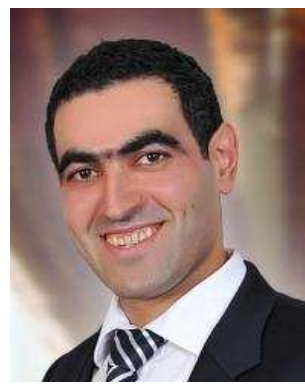

Mohammed El-Hajjar is a lecturer in the Electronics and Computer Science in the University of Southampton. He received his BEng degree in Electrical Engineering from the American University of Beirut, Lebanon in 2004. He then received an MSc in Radio Frequency Communication Systems and $\mathrm{PhD}$ in Wireless Communications both from the University of Southampton, UK in 2005 and 2008, respectively. Following the $\mathrm{PhD}$, he joined Imagination Technologies as a research engineer, where he worked on designing and developing the BICM peripherals in Imagination's multistandard communications platform, which resulted in several patent applications. In January 2012, he joined the Electronics and Computer Science in the University of Southampton as a lecturer in the Communications, Signal Processing and Control research group. He is the recipient of several academic awards and has published a Wiley-IEEE book and in excess of 30 journal and international conference papers. His research interests are mainly in the development of intelligent communications systems for the Internet of Things including massive MIMO systems for mm-wave communications, cooperative communications and Radio over fibre systems. 


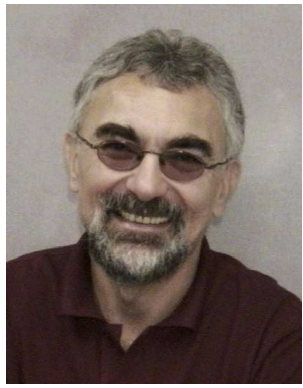

Lajos Hanzo (http://www-

mobile.ecs.soton.ac.uk) FREng, FIEEE, FIET, Fellow of EURASIP,

DSc received his degree in electronics in 1976 and his doctorate in 1983. In 2009 he was awarded the honorary doctorate "Doctor Honoris Causa" by the Technical University of Budapest. During his 35-year career in telecommunications he has held various research and academic posts in Hungary, Germany and the UK. Since 1986 he has been with the School of Electronics and Computer Science, University of Southampton, UK, where he holds the chair in telecommunications. He has successfully supervised $80 \mathrm{PhD}$ students, co-authored 20 John Wiley/IEEE Press books on mobile radio communications totalling in excess of 10000 pages, published 1300 research entries at IEEE Xplore, acted both as TPC and General Chair of IEEE conferences, presented keynote lectures and has been awarded a number of distinctions. Currently he is directing a 100-strong academic research team, working on a range of research projects in the field of wireless multimedia communications sponsored by industry, the Engineering and Physical Sciences Research Council (EPSRC) UK, the European IST Programme and the Mobile Virtual Centre of Excellence (VCE), UK. He is an enthusiastic supporter of industrial and academic liaison and he offers a range of industrial courses. He is also a Governor of the IEEE VTS. During 2008 - 2012 he was the Editor-in-Chief of the IEEE Press and since 2009 he has been a Chaired Professor also at Tsinghua University, Beijing. For further information on research in progress and associated publications please refer to http://www-mobile.ecs.soton.ac.uk 